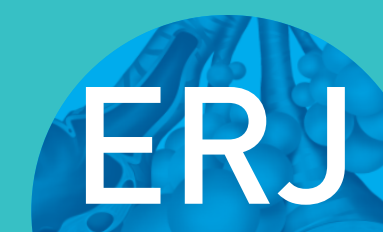

open research
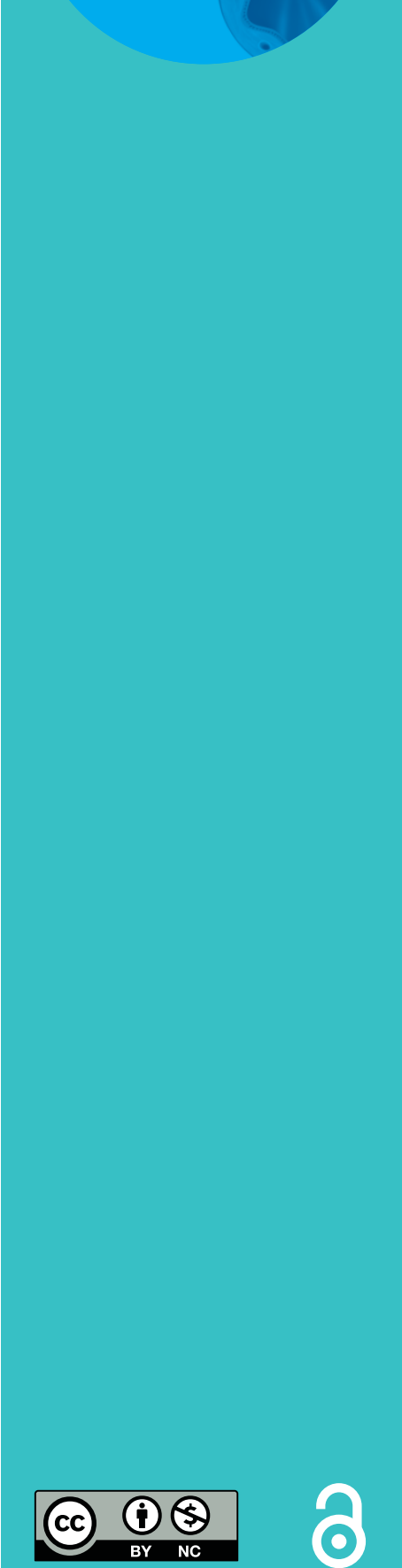

\section{Distal airway dysfunction identifies pulmonary inflammation in asymptomatic smokers}

\author{
Kenneth I. Berger ${ }^{1,2}$, Deepak R. Pradhan ${ }^{1,2}$, Roberta M. Goldring ${ }^{1,2}$, \\ Beno W. Oppenheimer ${ }^{1,2}$, William N. Rom ${ }^{2}$ and Leopoldo N. Segal ${ }^{1,2}$
}

Affiliations: ${ }^{1}$ André Cournand Pulmonary Laboratory, Bellevue Hospital Center, New York, NY, USA. ${ }^{2}$ Division of Pulmonary, Critical Care and Sleep Medicine, Dept of Medicine, New York University School of Medicine, New York, NY, USA.

Correspondence: Leopoldo N. Segal, NYU School of Medicine, 462 First Ave 7W54, New York, NY 10016, USA. E-mail: Leopoldo.Segalanyumc.org

ABSTRACT Smoking induced inflammation leads to distal airway destruction. However, the relationship between distal airway dysfunction and inflammation remains unclear, particularly in smokers prior to the development of airway obstruction.

Seven normal controls and 16 smokers without chronic obstructive pulmonary disease (COPD) were studied. Respiratory function was assessed using the forced oscillation technique (FOT). Abnormal FOT was defined as elevated resistance at $5 \mathrm{~Hz}(R 5)$. Parameters reflecting distal lung function included frequency dependence of resistance (R5-20) and dynamic elastance (X5). Inflammation was quantified in concentrated bronchoalveolar lavage utilising cell count differential and cytokines expressed as concentration per $\mathrm{mL}$ epithelial lining fluid.

All control subjects and seven smokers had normal R5. Nine smokers had elevated $R 5$ with abnormal R5-20 and $X 5$, indicating distal lung dysfunction. The presence of abnormal FOT was associated with twofold higher lymphocyte and neutrophil counts $(\mathrm{p}<0.025)$ and with higher interleukin (IL)-8, eotaxin and fractalkine levels $(\mathrm{p}<0.01)$. Reactivity of $R 5-20$ and $X_{5}$ correlated with levels of IL-8, eotaxin, fractalkine, IL-12p70 and transforming growth factor- $\alpha(r>0.47, p<0.01)$.

Distal airway dysfunction in smokers without COPD identifies the presence of distal lung inflammation that parallel reported observations in established COPD. These findings were not evident on routine pulmonary function testing and may allow the identification of smokers at risk of progression to COPD.

@ERSpublications

Isolated dysfunction in distal airways identifies pulmonary inflammation in asymptomatic smokers with normal airflow http://ow.ly/8bVk305aVkj

This article has supplementary material available from openres.ersjournals.com

Received: June 032016 | Accepted after revision: Oct 102016

Support statement: This study was supported by US National Institute for Occupational Safety and Health grant 200-2011-39413 and US National Institutes of Health grants K23 AI102970 and UL1 TR000038. Funding information for this article has been deposited with the Open Funder Registry.

Conflict of interest: Disclosures can be found alongside this article at openres.ersjournals.com

Copyright $\odot$ ERS 2016. This article is open access and distributed under the terms of the Creative Commons Attribution Non-Commercial Licence 4.0. 


\section{Introduction}

The diagnosis of chronic obstructive pulmonary disease (COPD) is dependent on the identification of reduced airflow using spirometry (Global Initiative for Chronic Obstructive Lung Disease (GOLD) criteria). However, spirometry may be normal early in the disease process. Furthermore, the GOLD guidelines have removed the "at risk" stage 0 , since many smokers do not progress to COPD as defined by clinical and spirometric data. This may, in part, reflect the inability of spirometry to detect dysfunction in the distal lung where lung units are lost early in disease [1]. It is recognised that these airways represent a "quiet zone" where considerable disease might be present before airflow limitation is identifiable using standard pulmonary function assessment [2].

Multiple tests have been used to identify small airway abnormalities in smokers; however, the relationship with COPD pathogenesis remains unclear. This study is based on the hypothesis that if small airway dysfunction is an early manifestation of a disease process that may evolve to COPD, it should be associated with inflammation at the site of injury (e.g. the distal lung). To test this hypothesis small airway function and distal lung inflammation were evaluated in smokers who did not meet criteria for COPD. Investigation of immunological derangements associated with distal airway dysfunction in the setting of normal spirometry may provide insight into pathophysiological mechanisms present at disease onset.

\section{Methods}

This investigation utilised forced oscillation testing (FOT) and its response to bronchodilator in smokers who did not meet GOLD criteria for COPD, but had focal emphysematous changes found incidentally upon chest computed tomography (CT) screening. These subjects were selected because the anatomical abnormality found suggested potential onset of disease at an early stage when COPD was not present. In vivo measurements of cell and cytokine levels in epithelial lining fluid (ELF) were correlated with respiratory function as assessed using FOT.

\section{Subjects}

23 subjects (seven normal controls and 16 smokers) underwent pulmonary function evaluation and bronchoscopy. Normal controls were asymptomatic nonsmokers $(<2$ pack-years) without history of lung disease. Smokers were enrolled from our lung cancer screening cohort.

Although all smokers had radiographic evidence for emphysema, findings were generally focal and mild in severity without hyperinflation. Post-bronchodilator spirometry revealed forced expiratory volume in $1 \mathrm{~s}$ (FEV1)/forced vital capacity (FVC) $\geqslant 70 \%$ in all but three subjects where values were $67-68 \%$. In addition, based on absence of symptoms, none of these subjects met GOLD criteria for COPD. The smokers were divided into smoker normal FOT and smoker abnormal FOT groups, based on presence of abnormal oscillometry without knowledge of measured inflammatory cytokines. Exclusion criteria were interstitial lung disease, lung nodules, use of inhaled or oral steroids, regular use of anti-inflammatory drugs, malignancy, significant hepatic, renal or cardiovascular disease, diabetes mellitus and alcohol use. Medical history and details of medication use and respiratory symptoms were obtained. Participants provided signed informed consent and the study was approved by the institutional review board of New York University and Bellevue Hospital (New York, NY, USA).

\section{Respiratory physiology}

Spirometry, plethysmography and diffusing capacity (Vmax; SensorMedics, Yorba Linda, CA, USA) were performed according to published guidelines [3]. Data were compared with published normative values [4-6].

All subjects underwent FOT (pre- and post-bronchodilator) using the Jaeger Impulse Oscillation System (Jaeger USA; Yorba Linda, CA, USA) during tidal breathing with support of the cheeks [7]. Trials with stable tidal and end-expiratory volume were analysed. Since 150 impulses are analysed over a 30-s measurement, coherence $>0.7$ at $5 \mathrm{~Hz}$ and $>0.85$ at $10 \mathrm{~Hz}$ were required [8]. Reproducibility between trials (variability $<10 \%$ ) was required.

FOT parameters included resistance at oscillating frequencies of $5 \mathrm{~Hz}\left(R_{5}\right)$ and $20 \mathrm{~Hz}\left(R_{2}\right)$, frequency dependence of resistance between 5 and $20 \mathrm{~Hz}(R 5-20)$, dynamic elastance as assessed by reactance at $5 \mathrm{~Hz}\left(X_{5}\right)$, reactance area and resonant frequency. The smoker normal FOT and smoker abnormal FOT groups were defined based on a global FOT parameter of respiratory resistance $(R 5)$. Conservative limits of normal were selected that approximate $150 \%$ of mean values in normal subjects [9-14]. Data obtained in our laboratory in 80 asymptomatic nonsmoking subjects with normal spirometry and without lung disease fell within these limits.

\section{In vivo distal lung inflammation}

All subjects underwent nasal bronchoscopy 1 week after pulmonary function testing. The bronchoscope was passed without suctioning until wedged in the lingula and right middle lobe sequentially for 
bronchoalveolar lavage (BAL). $150 \mathrm{~mL}$ of sterile saline was injected for each lavage. Cell count and differential from the BAL fluid was obtained after centrifugation. 39 cytokines were measured in concentrated BAL using Luminex with Human Cytokine Panel I (Millipore, Billerica, MA, USA). Since analytes in ELF are diluted with sterile saline during BAL, a concentration step was performed via dialysis and lyophilisation, using albumin as an internal control. After $\sim 75$-fold concentration, all six immunoglobulins and 28 out of 39 cytokines were within measurable levels. Cytokines levels that were below detectable limits were not included in the analyses (interferon (IFN)- $\alpha$, IFN- $\beta$, interleukin (IL)-10, IL-13, IL-17, IL-2, IL-3, IL-4 and tumour necrosis factor- $\beta$ ). Urea levels in BAL and plasma were used to calculate the concentration of cells, immunoglobulins and cytokines in ELF [15].

\section{Statistical analyses}

Data are presented as median (interquartile range). Comparisons among groups were made using the Kruskal-Wallis test, with subsequent post hoc Mann-Whitney U-testing between pairs. Statistical correlation of FOT parameters and inflammation was assessed via Pearson correlation after log-transforming inflammatory cytokine and immunoglobulin data. p-values $<0.05$ were considered statistically significant. Since obesity has been shown to have an effect on FOT parameters [10], we performed multivariate linear regression analysis to adjust data for body mass index (BMI). Where appropriate, adjustment for multiple comparisons was performed using the false discovery rate procedure [16, 17]. SPSS Statistics (version 20.0; IBM, Armonk, NY, USA) was used for all analyses.

\section{Results}

Subject characteristics

Clinical characteristics of the three subject groups are shown in table 1. The normal control group was younger than either of the smoker groups $(\mathrm{p}=0.003)$ and, by design, had no smoking history. The smoker normal FOT group did not differ from the smoker abnormal FOT group with respect to demographics, smoking history, symptoms or inhaler use. None of the smokers had respiratory symptoms consistent with chronic bronchitis (daily cough with sputum production for 3 months in two consecutive years).

Table 2 shows results of pulmonary function evaluation in the three groups. The normal control group demonstrated normal spirometry, lung volumes, plethysmographic resistance and diffusing capacity. The smoker abnormal FOT group demonstrated lower pre-bronchodilator FEV1/FVC compared with the normal control group; this difference was not apparent post-bronchodilator. In addition, both smoker groups demonstrated higher values for total lung capacity compared with the normal control group, but the measured values remained in the normal range. Comparison between the two smoker subgroups showed trends for difference in median values for pre-bronchodilator FEV1 and specific conductance that did not reach statistical significance, since the interquartile ranges were similar. Thus, differences were not demonstrable between the smoker subgroups with respect to pre- or post-bronchodilator spirometry, lung volumes, plethysmographic resistance or diffusing capacity.

\section{TABLE 1 Subject demographics and characteristics}

\begin{tabular}{|c|c|c|c|}
\hline & Normal control & Smoker normal FOT & Smoker abnormal FOT \\
\hline Subjects & 7 & 7 & 9 \\
\hline \multicolumn{4}{|l|}{ Demographic data } \\
\hline Age years & $35(28-49)$ & $61(58-68)^{*}$ & $63(60-67)^{*}$ \\
\hline Male & $6(86)$ & $6(86)$ & $5(56)$ \\
\hline Caucasian & $5(71)$ & $7(100)$ & $9(100)$ \\
\hline $\mathrm{BMI} \mathrm{kg} \cdot \mathrm{m}^{-2}$ & $28(21-30)$ & $31(25-33)$ & $27(25-29)$ \\
\hline \multicolumn{4}{|l|}{ Tobacco use } \\
\hline Current smoker & $0(0)$ & $1(14)$ & 3 (33) \\
\hline Duration pack-years & $0(0)$ & $31(15-46)^{*}$ & $46(31-49)^{*}$ \\
\hline \multicolumn{4}{|l|}{ Symptoms } \\
\hline Any symptom & $0(0)$ & $1(14)$ & $2(22)$ \\
\hline Exertional dyspnoea & $0(0)$ & $1(14)$ & $1(11)$ \\
\hline Wheeze & $0(0)$ & $1(14)$ & $1(11)$ \\
\hline Cough & $0(0)$ & $0(0)$ & $2(22)$ \\
\hline Sputum production & $0(0)$ & $0(0)$ & $1(11)$ \\
\hline
\end{tabular}




\begin{tabular}{|c|c|c|c|}
\hline & Normal control & Smoker normal FOT & Smoker abnormal FOT \\
\hline Subjects & 7 & 7 & 9 \\
\hline \multicolumn{4}{|l|}{ Pre-bronchodilator spirometry } \\
\hline FVC \% pred & 100 (92-105) & $98(93-111)$ & $96(85-102)$ \\
\hline FEV $1 \%$ pred & $95(86-101)$ & $99(89-106)$ & $84(79-95)$ \\
\hline $\mathrm{FEV}_{1} / \mathrm{FVC}$ & $79(76-84)$ & $74(69-75)$ & $72(65-77)^{*}$ \\
\hline \multicolumn{4}{|l|}{ Post-bronchodilator spirometry } \\
\hline FVC \% pred & 102 (96-107) & $96(89-114)$ & $96(91-101)$ \\
\hline $\mathrm{FEV}_{1} \%$ pred & $100(84-104)$ & $97(94-111)$ & $93(81-102)$ \\
\hline FEV $1 / F V C$ & 83 (79-89) & $74(71-79)$ & $72(71-83)$ \\
\hline \multicolumn{4}{|l|}{ Plethysmography } \\
\hline \multicolumn{4}{|l|}{ Lung volumes } \\
\hline TLC \% pred & $85(80-88)$ & 97 (87-129)* & 103 (99-107)* \\
\hline \multicolumn{4}{|l|}{ Resistance } \\
\hline Raw $\mathrm{cmH}_{2} \mathrm{O} \cdot \mathrm{L}^{-1} \cdot \mathrm{s}^{-1}$ & $1.43(1.23-1.60)$ & $1.54(1.22-2.01)$ & $2.19(1.16-2.30)$ \\
\hline $\mathrm{s} G$ aw $\mathrm{L} \cdot \mathrm{S}^{-1} \cdot \mathrm{cmH}_{2} \mathrm{O}^{-1} \cdot \mathrm{L}^{-1}$ & $0.20(0.83-0.21)$ & $0.19(0.15-0.23)$ & $0.12(0.10-0.21)$ \\
\hline \multicolumn{4}{|l|}{ Diffusing capacity } \\
\hline$D \mathrm{LCO} / V \mathrm{~A} \mathrm{~mL} \cdot \mathrm{min}^{-1} \cdot \mathrm{mmHg}^{-1} \cdot \mathrm{L}^{-1}$ & $4.87(4.44-5.27)$ & $4.06(3.56-4.97)$ & $3.44(3.18-3.92)$ \\
\hline \multicolumn{4}{|l|}{ FOT } \\
\hline \multicolumn{4}{|l|}{ Resistance } \\
\hline$R 5 \mathrm{kPa} \cdot \mathrm{L}^{-1} \cdot \mathrm{s}^{-1}$ & $0.36(0.29-0.38)$ & $0.33(0.31-0.34)$ & $0.57(0.45-0.59)^{*, \#}$ \\
\hline$R_{20} \mathrm{kPa} \cdot \mathrm{L}^{-1} \cdot \mathrm{s}^{-1}$ & $0.31(0.27-0.34)$ & $0.28(0.25-0.30)$ & $0.40(0.35-0.47)^{*, \#}$ \\
\hline$R 5-20 \mathrm{kPa} \cdot \mathrm{L}^{-1} \cdot \mathrm{s}^{-1}$ & $0.05(0.03-0.06)$ & $0.05(0.03-0.07)$ & $0.12(0.05-0.18)^{* . \#}$ \\
\hline \multicolumn{4}{|l|}{ Reactance } \\
\hline $\mathrm{AX} \mathrm{kPa} \cdot \mathrm{L}^{-1}$ & $0.23(0.13-0.45)$ & $0.21(0.13-0.39)$ & $0.72(0.43-1.82)^{*, \#}$ \\
\hline$X_{5} \mathrm{kPa} \cdot \mathrm{L}^{-1} \cdot \mathrm{s}^{-1}$ & $-0.10(-0.13-0.07)$ & $-0.10(-0.12-0.05)$ & $-0.16(-0.25-0.13)^{* . \#}$ \\
\hline fres $\mathrm{Hz}$ & $12.6(9.3-14.2)$ & $12.3(9.6-14.3)$ & $18.7(13.2-22.9)^{*, \#}$ \\
\hline \multicolumn{4}{|c|}{ 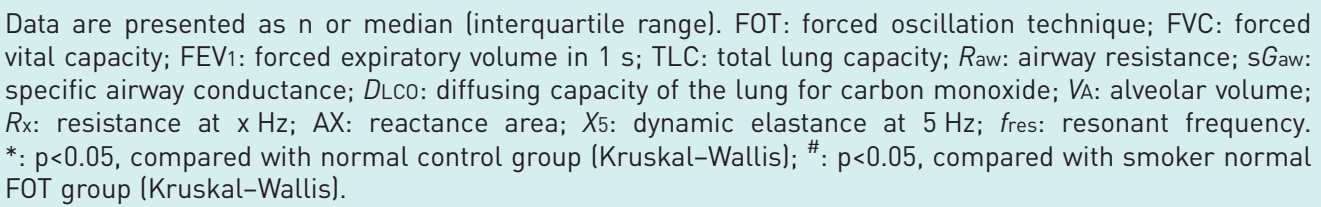 } \\
\hline
\end{tabular}

In addition, table 2 shows results for oscillometry measurements. For the normal control group, all FOT parameters were within the normal range [9-13]. The smoker normal FOT group also demonstrated normal values for resistance and reactance parameters that did not differ from the normal control group. In contrast, while the smoker abnormal FOT group was defined by an abnormal $R 5$, abnormalities were also noted in $R_{5-20}$ and $X_{5}$, indicating dysfunction in the lung periphery that was not evident on spirometry.

Figure 1 shows pre- and post-bronchodilator measurements of $\mathrm{FEV} 1, R 5, R_{5}-20$ and $X_{5}$ for all subjects within each group. The FEV1 response to bronchodilator was variable within subjects in all three groups. The normal control and smoker normal FOT groups did not demonstrate a change in FEV1 post-bronchodilator. A statistically significant increase in FEV1 was noted in the smoker abnormal FOT group, but an increase $>12 \%$ was evident in only a single individual. The normal control and smoker normal FOT groups did not demonstrate bronchodilator response of any of the FOT parameters. However, the smoker abnormal FOT group demonstrated significant bronchodilator response for all FOT parameters $\left(R 5 \mathrm{p}=0.011 ; X_{5} \mathrm{p}=0.008\right.$; $R 5-20 \mathrm{p}=0.02)$. FOT parameters remained abnormal in approximately half of this subgroup.

\section{In vivo distal lung inflammation}

The relationship between presence of distal airway dysfunction and lower airway inflammation is illustrated in figures 2 and 3. Figure 2 illustrates neutrophil and lymphocyte cell counts expressed as concentration per mL ELF. Inflammatory cell counts did not differ between normal control and smoker normal FOT groups. However, the smoker abnormal FOT group had more than two-fold higher median neutrophil and lymphocyte cell counts, compared to the smoker normal FOT group (2.5 (1.7-3.9) versus $1.3(1.2-1.6) \times 10^{5}$ cells $\cdot \mathrm{mL}^{-1}, \mathrm{p}=0.016$ versus $10.1(5.9-16.4)$ versus $3.7(1.3-6.8) \times 10^{5}$ cells $\cdot \mathrm{mL}^{-1}, \mathrm{p}=0.022$, respectively). Levels of three chemoattractant cytokines (IL-8, eotaxin and fractalkine) were significantly higher in the smoker abnormal FOT group compared to the other groups with normal distal airway function (figure 3, p<0.01 for all comparisons). Table 3 shows the correlations that were statistically significant (after adjustment for multiple comparisons) between inflammatory markers and spirometry 

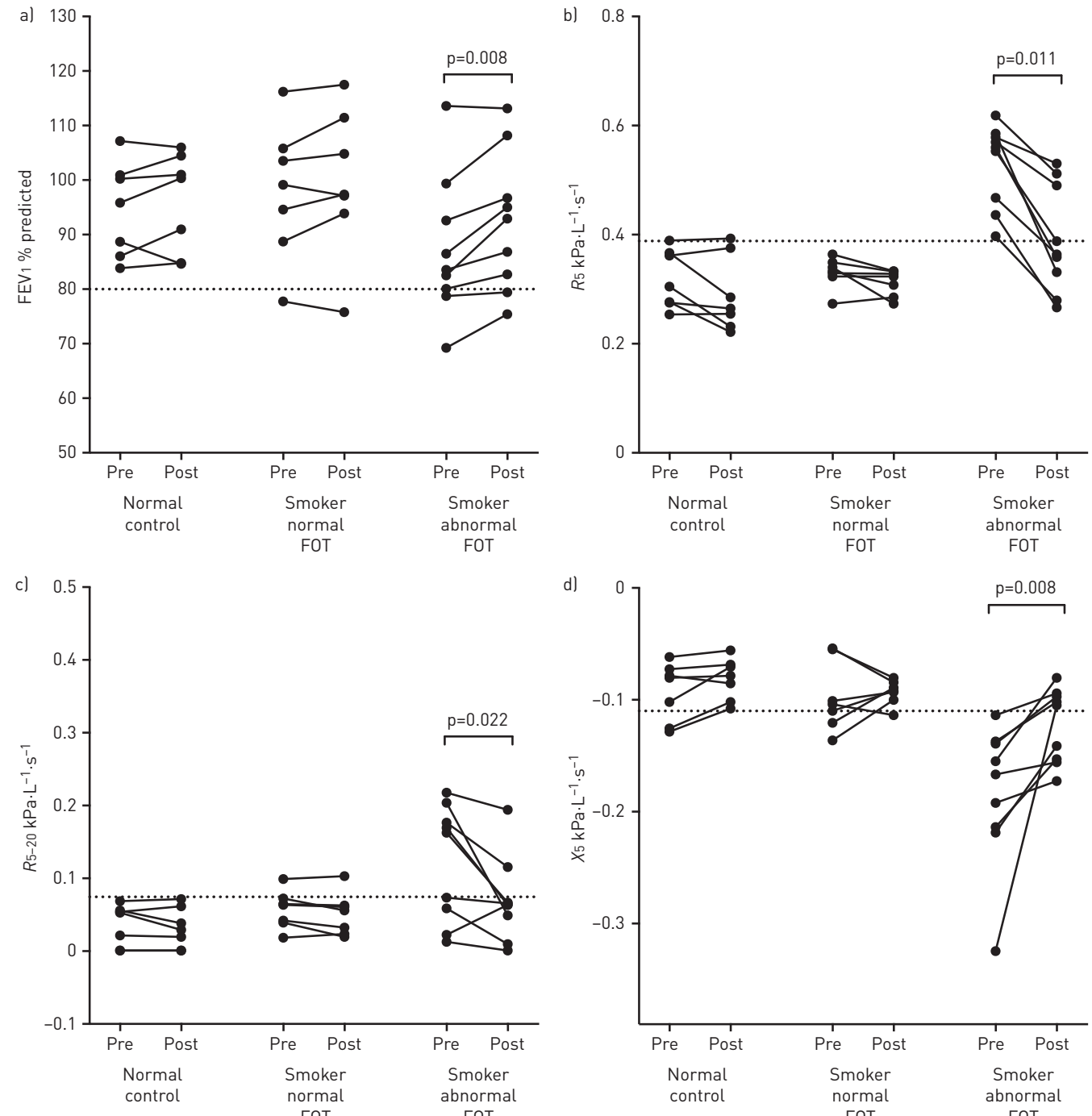

FIGURE 1 Comparison of pre- and post-bronchodilator data for spirometric and oscillometric parameters. Dotted lines denote the limits of normal. Paired comparisons revealed that the normal control and smoker normal forced oscillation technique (FOT) groups did not demonstrate bronchodilator response of either forced expiratory volume in $1 \mathrm{~s}$ ( $\left.F E V_{1}\right)$ or any of the FOT parameters. a) A statistically significant increase in FEV1 was noted in the smoker abnormal FOT group, but an increase $>12 \%$ was evident in only a single individual. In addition, the smoker abnormal FOT group demonstrated significant bronchodilator response for b) resistance at $5 \mathrm{~Hz}(R 5 ; \mathrm{p}=0.011), c)$ dynamic elastance as assessed by reactance at $5 \mathrm{~Hz}\left(X_{5} ; \mathrm{p}=0.008\right)$ and d) frequency dependence of resistance $(R 5-20 ; p=0.022)$.

and FOT parameters (full correlation matrix is available in online supplementary table S1). A statistically significant correlation with FEV1 was not evident for lymphocytes or neutrophils and was only present for IL-12 p70. R20 correlated with neutrophils but not with any chemokine. In contrast, FOT markers of distal airway function (R5-20 and/or X5) correlated with levels of neutrophils, eotaxin, IL-8, fractalkine, IL-12 p70, macrophage inflammatory protein- $1 \alpha$ and growth-regulated oncogene.

\section{Airway reactivity and distal lung inflammation}

Airways responsiveness has been proposed as an important factor in COPD pathogenesis [18]. Therefore, we evaluated the correlation between magnitude of airway reactivity and degree of in vivo distal lung inflammation. Table 4 shows the statistically significant correlations (after adjustment for multiple comparisons) between inflammatory markers and bronchodilator response when assessed for spirometry and FOT parameters (full correlation matrix is presented in online supplementary table S2). The bronchodilator response FEV1 or R20 did not correlate with either inflammatory cell counts or chemokine levels. In contrast, the bronchodilator response of $R_{5-20}$ and $X_{5}$ were correlated with levels of five chemokines in ELF. These correlations remained significant in multivariate models that included BMI and 

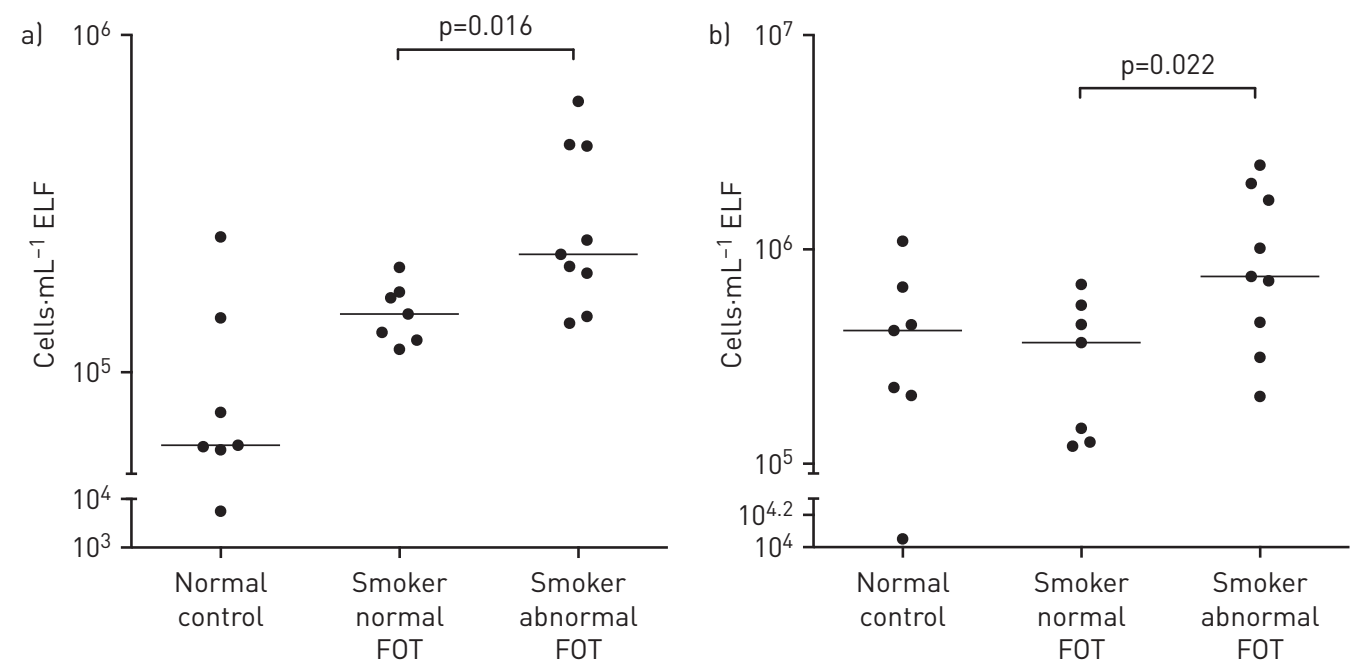

FIGURE 2 Evaluation of inflammatory cell count in the three groups. Cell counts in epithelial lining fluid (ELF) were plotted on a logarithmic scale for the normal control, smoker normal forced oscillation technique (FOT) and smoker abnormal FOT groups. a) Neutrophil and b) lymphocyte counts were higher in the smoker abnormal FOT group compared with both the smoker normal FOT group ( $p=0.016$ and 0.022 , respectively) and the normal control group ( $\mathrm{p}=0.009$ and 0.021 , respectively).

baseline lung function. Correlations were observed for chemokines reflecting multiple immunological pathways including chemoattractant cytokines (IL-8, eotaxin and fractalkine), growth factors (transforming growth factor- $\alpha$ ), and T-helper (Th) type 1 cytokines (IL-12 p70). A representative example (IL-8) is illustrated in figure 4. A clear separation is visible between data obtained in the smoker abnormal FOT group, compared with the other subject groups.

\section{Discussion}

This study demonstrates that in asymptomatic smokers a distinct physiological behaviour, consistent with distal lung dysfunction, is associated with subclinical distal lung inflammation. Despite presence of emphysema, these findings occurred at a point during disease progression when post-bronchodilator spirometry remained normal and subjects were asymptomatic and, therefore, a clinical diagnosis of COPD could not be established using GOLD criteria [19]. The pattern of inflammation indicated activation of innate and cellular immune responses. Furthermore, the elevated inflammatory biomarkers in these asymptomatic subjects with distal lung dysfunction have been shown to predict disease progression in patients with established COPD [20,21]. Thus, investigation of distal lung mechanics in asymptomatic smokers may identify a subgroup of subjects at risk for progression to COPD.
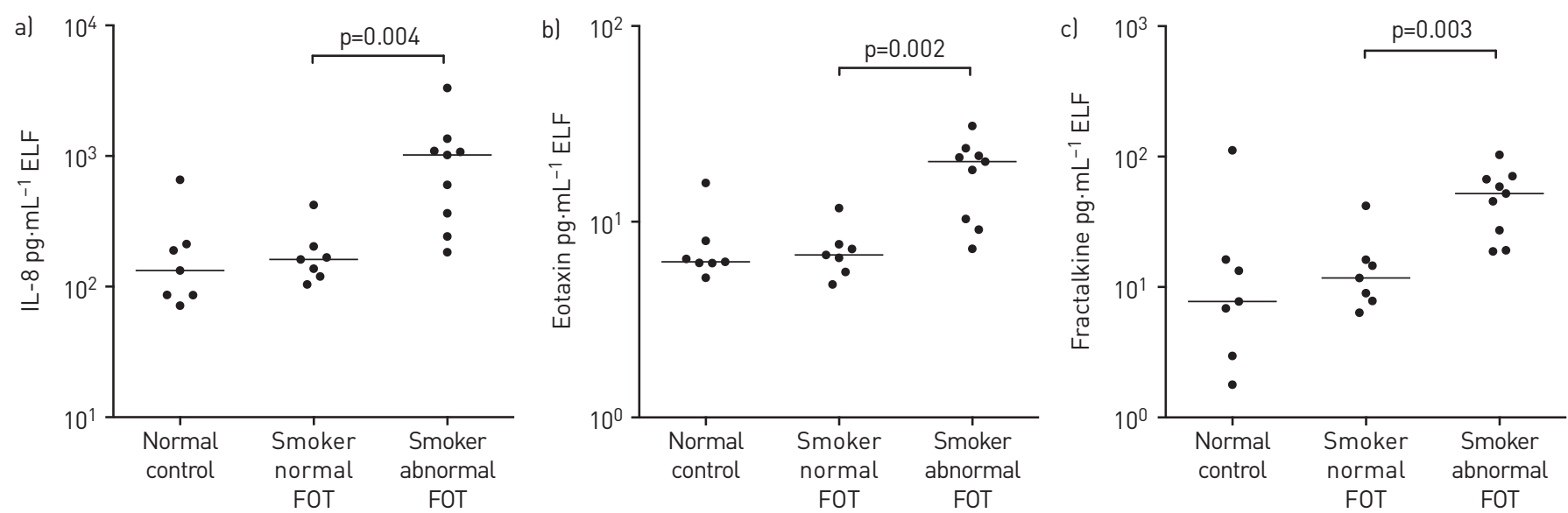

FIGURE 3 Concentration of inflammatory cytokines in epithelial lining fluid (ELF) were plotted for the normal control, smoker normal forced oscillation technique (FOT), and smoker abnormal FOT groups. Statistically higher levels of a) interleukin (IL)-8; b) eotaxin; and c) fractalkine were noted in the smoker abnormal FOT group, compared with the smoker normal FOT group ( $p=0.004,0.002$ and 0.003 , respectively). 


\section{TABLE 3 Correlation between in vivo inflammation and lung function}

\begin{tabular}{|c|c|c|c|c|c|c|c|c|}
\hline & \multicolumn{2}{|c|}{ FEV 1} & \multicolumn{2}{|c|}{$R_{20}$} & \multicolumn{2}{|c|}{$R 5-20$} & \multicolumn{2}{|c|}{$X_{5}$} \\
\hline & $\rho$ & p-value & $\rho$ & p-value & $\rho$ & p-value & $\rho$ & p-value \\
\hline \multicolumn{9}{|c|}{ Inflammatory cells } \\
\hline Neutrophils & -0.42 & 0.045 & 0.51 & $0.015^{\#}$ & 0.55 & $0.007^{\#}$ & -0.55 & $0.007^{\#}$ \\
\hline \multicolumn{9}{|l|}{ Chemokines } \\
\hline Eotaxin & -0.49 & 0.019 & 0.56 & 0.006 & 0.48 & 0.022 & -0.77 & $<0.001^{\#}$ \\
\hline IL-8 & -0.34 & NS & 0.51 & 0.013 & 0.51 & 0.012 & -0.69 & $<0.001^{\#}$ \\
\hline Fractalkine & -0.31 & NS & 0.42 & 0.045 & 0.40 & NS & -0.56 & $0.006^{\#}$ \\
\hline IL-12 p70 & -0.65 & $0.001^{\#}$ & 0.37 & NS & 0.49 & 0.018 & -0.55 & $0.006^{\#}$ \\
\hline MIP- $1 \alpha$ & -0.57 & 0.005 & 0.39 & NS & 0.58 & $0.004^{\#}$ & -0.54 & $0.008^{\#}$ \\
\hline GRO & -0.34 & NS & 0.25 & NS & 0.73 & $<0.001^{\#}$ & -0.46 & 0.029 \\
\hline
\end{tabular}

FEV1: forced expiratory volume in $1 \mathrm{~s}$; $R \mathrm{x}$ : resistance at $\mathrm{x} \mathrm{Hz}$; $\mathrm{X}_{5}$ : dynamic elastance at $5 \mathrm{~Hz}$; IL: interleukin; MIP: macrophage inflammatory protein; GRO: growth-regulated oncogene; NS: nonsignificant. \#: statistically significant after correction for multiple comparisons.

In COPD, lung damage starts in airways of small calibre, producing irreversible tissue destruction and airflow obstruction $[22,23]$. Micro-CT of surgical specimens from GOLD stage I patients showed airway narrowing and loss of small conducting airways [24]. However, the contribution of these distal airways to total resistance is minimal and detection of early abnormalities by routine spirometry is limited [25]. Therefore, the spirometric abnormalities required for the diagnosis of COPD occur only later in the course of disease. Based on these considerations, we evaluated FOT markers of distal lung function in smokers who might be at risk for progression to COPD, in accordance with renewed interest in distal lung mechanics in patients with established COPD [26-28].

It has been suggested that abnormal distal lung function might identify clinically relevant phenotypes of COPD [26]. Thus, we investigated the correlation between functional abnormalities of the distal lung and inflammatory biomarkers in the distal lung. In this study, a subgroup of smokers demonstrated physiological abnormalities characterised by frequency dependence of resistance and abnormal dynamic elastance. Frequency dependence of resistance correlates with frequency dependence of compliance and may reflect focal areas of increased small airway impedance with resultant nonuniform distribution of airflow [29]. Although upper airway shunt, glottis closure and chest wall abnormalities may also produce frequency dependence of resistance, these factors cannot explain the observed bronchodilator responsiveness of FOT parameters. Moreover, the correlation with increased subclinical inflammation in the distal lung reinforces our interpretation that the pattern of FOT abnormality reflects distal lung dysfunction. Of importance, this correlation between functional abnormality and inflammation required investigation of distal lung function beyond that which is obtainable from routine evaluation (spirometry, lung volumes and diffusion).

The importance of bronchial responsiveness in COPD pathogenesis has been a matter of debate since the Dutch hypothesis was introduced in 1961 [18]. By convention, responsiveness of spirometric parameters has been the major tool used to investigate airway reactivity. This study extends prior observations by

TABLE 4 Correlation between in vivo inflammation and physiological bronchodilator response

\begin{tabular}{|c|c|c|c|c|c|c|c|c|}
\hline & \multicolumn{2}{|c|}{ FEV $1 \%$ change } & \multicolumn{2}{|c|}{$\Delta R_{20}$} & \multicolumn{2}{|c|}{$\Delta R \mathbf{5}-20$} & \multicolumn{2}{|c|}{$\Delta X_{5}$} \\
\hline & $\rho$ & p-value & $\rho$ & p-value & $\rho$ & p-value & $\rho$ & p-value \\
\hline \multicolumn{9}{|c|}{ Inflammatory cells } \\
\hline Neutrophils & 0.02 & NS & 0.09 & NS & -0.30 & NS & 0.40 & NS \\
\hline \multicolumn{9}{|l|}{ Chemokines } \\
\hline IL-8 & 0.12 & NS & 0.04 & NS & -0.51 & 0.021 & 0.71 & $<0.001^{\#}$ \\
\hline Eotaxin & 0.26 & NS & -0.13 & NS & -0.52 & 0.016 & 0.66 & $0.001^{\#}$ \\
\hline Fractalkine & 0.28 & NS & -0.02 & NS & -0.58 & 0.006 & 0.65 & $0.001^{\#}$ \\
\hline IL-12 p70 & 0.28 & NS & -0.03 & NS & -0.47 & 0.032 & 0.56 & $0.008^{\#}$ \\
\hline TGF- $\alpha$ & 0.26 & NS & -0.06 & NS & -0.39 & NS & 0.56 & $0.008^{\#}$ \\
\hline
\end{tabular}

FEV1: forced expiratory volume in $1 \mathrm{~s} ; R \mathrm{x}$ : resistance at $\mathrm{x} \mathrm{Hz}$; $\mathrm{X}_{5}$ : dynamic elastance at $5 \mathrm{~Hz}$; IL: interleukin; TGF: transforming growth factor; NS: nonsignificant. \#: statistically significant after correction for multiple comparisons. 

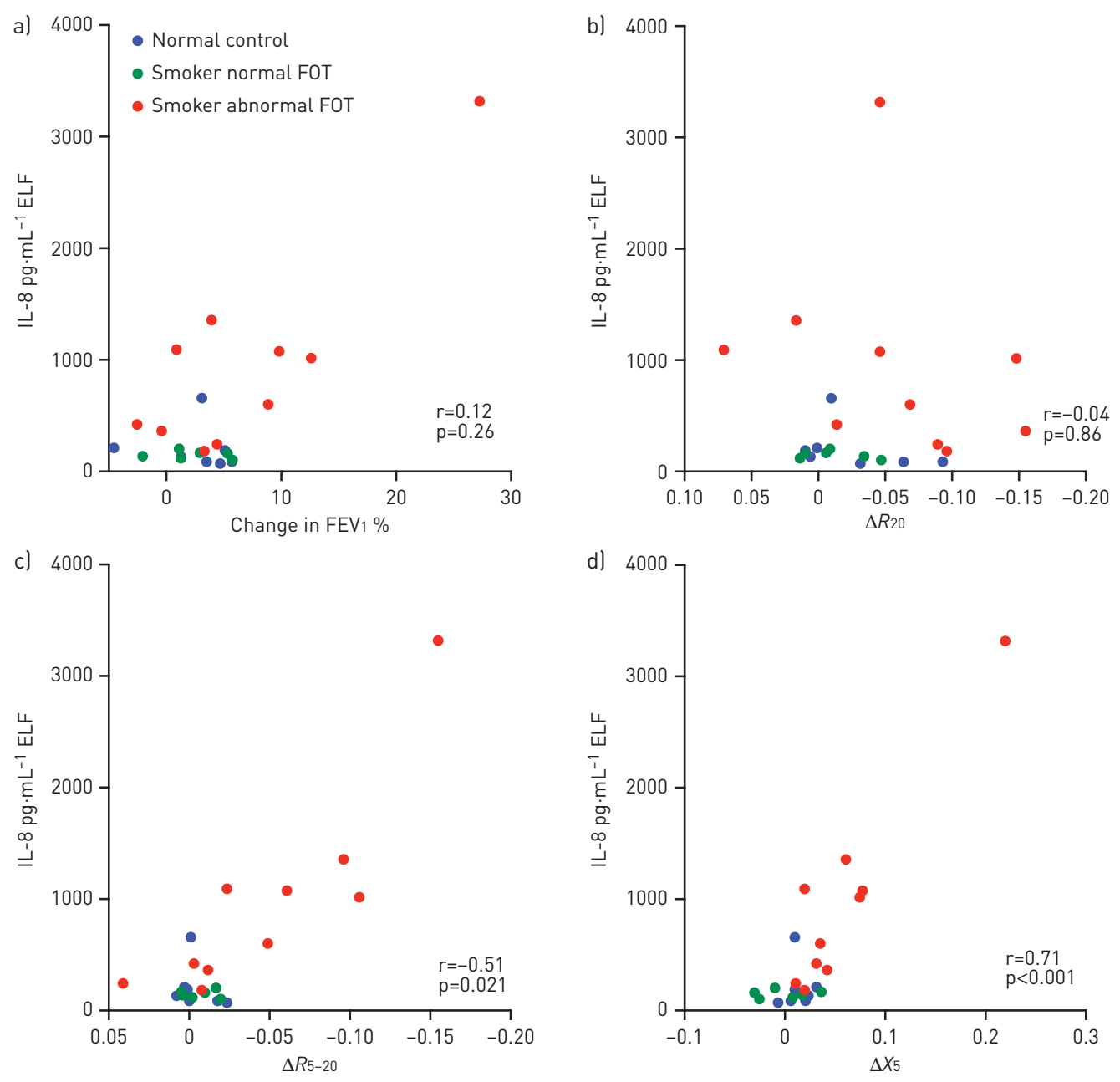

FIGURE 4 Relationship between bronchodilator response of physiological parameters and concentration of interleukin (IL)-8 in epithelial lining fluid (ELF). Bronchodilator response of al forced expiratory volume in $1 \mathrm{~s}$ (FEV 1$)$ and b) resistance at $20 \mathrm{~Hz}\left(R_{20}\right)$ did not correlate with the level of IL-8 in ELF. In contrast, the bronchodilator response of c) frequency dependence of resistance $(R 5-20)$ and d) dynamic elastance as assessed by reactance at $5 \mathrm{~Hz}\left(X_{5}\right)$ correlated with the level of $\mathrm{IL}-8$ in $E L F(r=-0.51, p=0.021$ and $r=0.71$, $\mathrm{p}<0.001$, respectively).

investigation of bronchodilator-elicited reactivity in the distal lung. Bronchodilator responsiveness of distal airway function ( $25-20$ and $X 5)$ was noted in all smokers with abnormal FOT. A predominant smooth muscle mechanism may explain return of distal airway function to the normal range while persistent abnormality suggests potential airway remodelling. In addition, the association between inflammatory markers and distal lung bronchodilator reactivity identifies a phenotype of increased distal lung inflammation. The presence of this correlation prior to the development of fixed obstruction on spirometry is in accord with onset of disease in the distal lung $[20,22]$. In addition, the correlation between the degree of distal airway reactivity and magnitude of distal lung inflammation supports a role for airway reactivity in COPD pathogenesis.

The pattern of inflammation observed in subjects with distal lung dysfunction was characterised by elevated inflammatory cells (neutrophils and lymphocytes) as well as elevated chemoattractants (IL-8 and eotaxin) and inflammatory cytokines (fractalkine). These findings are compatible with observations of neutrophilic inflammation in small airways of subjects with early COPD [20, 22] that leads to release of neutrophil elastase and parenchymal destruction [30]. A prior study has demonstrated elevated neutrophils, lymphocytes, IL-8 and eotaxin in sputum obtained from subjects with COPD [31]. In addition, in the present study, fractalkine was elevated, which may indicate activation of lymphocytes, dendritic cells and monocytes/macrophages $[32,33]$. Fractalkine is involved in differentiation of Th17 cells [32]. Importantly, levels of IL-17A and IL-22 are increased in sputum of individuals with COPD [34, 35], and Th17 cells have been identified in bronchial biopsies of patients with COPD [36]. Although earlier studies were able to identify subgroups within COPD cohorts with abnormal oscillometry [26-28], this study extends prior observations by correlating oscillometric abnormalities in asymptomatic smokers to in 
vivo lung inflammation at the site of injury (ELF). In our study, subjects with the most abnormal distal airway function demonstrated activation of innate and adaptive immune responses with a pattern that parallels observations from peripheral airway biopsies in subjects with established COPD [37].

Correlations observed between distal lung reactivity and inflammatory cytokines provide additional insight into disease pathogenesis. The correlations were independent of the baseline abnormality, highlighting that bronchodilator reactivity is an additional clinically relevant marker of distal lung inflammation. These associations included eotaxin, a well-established cytokine associated with bronchial reactivity [38]. Although elevated eosinophils were not observed, as might be expected in asthma [39], elevation of other neutrophilic chemoattractant and Th1 cytokines suggest a different mechanism for bronchodilator responsiveness in COPD. These observations indicate that, in asymptomatic smokers prior to COPD diagnosis using GOLD criteria, both baseline and bronchodilator behaviour of the distal lung are associated with activation of multiple innate immune pathways.

This study is a cross-sectional investigation of asymptomatic smokers with focal mild emphysema incidentally noted on CT performed for cancer screening. The size of the study cohort was limited by the complexity and invasive nature of the assessments. Despite the small number of subjects, a consistent association was demonstrated between physiological behaviour and in vivo inflammation. Progression to clinically evident COPD could not be evaluated and will require longitudinal investigation of larger cohorts. A difference in age was noted between the normal controls compared with the two smoker subgroups. However, the differences in inflammatory cells and mediators were noted in the smoker abnormal FOT group compared with the smoker normal FOT group, despite nearly identical ages. Furthermore, the observed abnormalities in these asymptomatic smokers could reflect concomitant asthma, bronchiolitis or respiratory infection. However, these alternative explanations are unlikely in the absence of clinical symptoms or recent exacerbations. Lastly, while it is biologically plausible to link inflammation with the observed physiological abnormalities, these correlations do not establish causality between these independent measures. Nevertheless, this study serves as a proof of concept, suggesting that the identification of a subset of asymptomatic smokers with a higher degree of lung inflammation may be relevant for developing earlier and more aggressive interventions aimed at halting or attenuating disease progression even before there is significant airflow obstruction or symptoms. Future trials with anti-inflammatory treatment might be warranted in this population.

Subjects were selected for study with the presence of focal mild emphysema on CT, but without COPD based on GOLD criteria. An incidental finding of emphysema is common in smokers [40]. Although the clinical significance of incidentally noted emphysema remains unclear, it was used for study entry since the anatomical abnormality suggests the potential onset of disease at an early stage when COPD was not present according to GOLD criteria. It should be noted that although the radiographic finding was localised to selected region(s), the oscillometry technique assesses global measures of the entire respiratory system, and the field sampled by the BAL technique generally did not correspond to the anatomical location of emphysema noted. These considerations suggest more widespread functional derangements than those demonstrated by the imaging data. The presence of distal airway dysfunction in these asymptomatic smokers with incidental findings of emphysema was associated with pulmonary inflammation despite absence of COPD according to GOLD criteria.

\section{Conclusions}

Two novel approaches were used to correlate distal airway function and inflammation in asymptomatic smokers prior to the development of fixed obstruction on spirometry. Abnormalities in distal airway function as assessed by oscillometric frequency dependence of resistance and reactance are associated with increased in vivo lung inflammation. These functional abnormalities were not identifiable on the basis of routine pulmonary function parameters. Thus, the present study demonstrates that distal lung dysfunction and bronchodilator reactivity are physiological expressions of a phenotype characterised by distal lung inflammation that parallels reported observations in established COPD.

\section{References}

1 Hogg JC, Chu F, Utokaparch S, et al. The nature of small-airway obstruction in chronic obstructive pulmonary disease. N Engl J Med 2004; 350: 2645-2653.

2 Mead J. The lung's “quiet zone". N Engl J Med 1970; 282: 1318-1319.

3 Miller MR, Crapo R, Hankinson J, et al. General considerations for lung function testing. Eur Respir J 2005; 26 : 153-161.

4 Hankinson JL, Odencrantz JR, Fedan KB. Spirometric reference values from a sample of the general U.S. population. Am J Respir Crit Care Med 1999; 159: 179-187.

5 Goldman HI, Becklake MR. Normal values at median altitudes and the prediction of normal results. Am Rev Tuberc 1959; 79: 457-467. 
6 Van Ganse WF, Ferris BG Jr, Cotes JE. Cigarette smoking and pulmonary diffusing capacity (transfer factor). Am Rev Respir Dis 1972; 105: 30-41.

7 Oostveen E, MacLeod D, Lorino H, et al. The forced oscillation technique in clinical practice: methodology, recommendations and future developments. Eur Respir J 2003; 22: 1026-1041.

8 Komarow HD, Myles IA, Uzzaman A, et al. Impulse oscillometry in the evaluation of diseases of the airways in children. Ann Allergy Asthma Immunol 2011; 106: 191-199.

9 Oppenheimer BW, Goldring RM, Herberg ME, et al. Distal airway function in symptomatic subjects with normal spirometry following World Trade Center dust exposure. Chest 2007; 132: 1275-1282.

10 Friedman SM, Maslow CB, Reibman J, et al. Case-control study of lung function in World Trade Center Health Registry area residents and workers. Am J Respir Crit Care Med 2011; 184: 582-589.

11 Goldman MD, Saadeh C, Ross D. Clinical applications of forced oscillation to assess peripheral airway function. Respir Physiol Neurobiol 2005; 148: 179-194.

12 Kohlhäufl M, Brand P, Scheuch G, et al. Impulse oscillometry in healthy nonsmokers and asymptomatic smokers: effects of bronchial challenge with methacholine. J Aerosol Med 2001; 14: 1-12.

13 Newbury W, Crockett A, Newbury J. A pilot study to evaluate Australian predictive equations for the impulse oscillometry system. Respirology 2008; 13: 1070-1075.

14 Oostveen E, Boda K, van der Grinten CP, et al. Respiratory impedance in healthy subjects: baseline values and bronchodilator response. Eur Respir J 2013; 42: 1513-1523.

15 Rennard SI, Basset G, Lecossier D, et al. Estimation of volume of epithelial lining fluid recovered by lavage using urea as marker of dilution. J Appl Physiol 1986; 60: 532-538.

16 Reiner A, Yekutieli D, Benjamini Y. Identifying differentially expressed genes using false discovery rate controlling procedures. Bioinformatics 2003; 19: 368-375.

17 Hochberg Y, Benjamini Y. More powerful procedures for multiple significance testing. Stat Med 1990; 9: 811-818.

18 Orie NGM, Sluiter HJ, De Vries K, et al. The host factor in bronchitis. In: Orie NGM, Sluiter HJ, eds. Bronchitis. Assen, Royal Van Gorcum, 1961; pp. 43-59.

19 Vestbo J, Hurd SS, Agustí AG, et al. Global strategy for the diagnosis, management and prevention of chronic obstructive pulmonary disease: GOLD Executive Summary. Am J Respir Crit Care Med 2013; 187: 347-365.

20 Cosio MG, Cosio Piqueras MG. Pathology of emphysema in chronic obstructive pulmonary disease. Monaldi Arch Chest Dis 2000; 55: 124-129.

21 Cosio MG, Saetta M, Agustí A. Immunologic aspects of chronic obstructive pulmonary disease. N Engl J Med 2009; 360: 2445-2454.

22 Cosio M, Ghezzo H, Hogg JC, et al. The relations between structural changes in small airways and pulmonary-function tests. N Engl J Med 1978; 298: 1277-1281.

23 Hogg JC, Macklem PT, Thurlbeck WM. Site and nature of airway obstruction in chronic obstructive lung disease. N Engl J Med 1968; 278: 1355-1360.

24 McDonough JE, Yuan R, Suzuki M, et al. Small-airway obstruction and emphysema in chronic obstructive pulmonary disease. $N$ Engl J Med 2011; 365: 1567-1575.

25 Pedley TJ, Schroter RC, Sudlow MF. The prediction of pressure drop and variation of resistance within the human bronchial airways. Respir Physiol 1970; 9: 387-405.

26 Crim C, Celli B, Edwards LD, et al. Respiratory system impedance with impulse oscillometry in healthy and COPD subjects: ECLIPSE baseline results. Respir Med 2011; 105: 1069-1078.

27 Di Mango AM, Lopes AJ, Jansen JM, et al. Changes in respiratory mechanics with increasing degrees of airway obstruction in COPD: detection by forced oscillation technique. Respir Med 2006; 100: 399-410.

28 Frantz S, Nihlén U, Dencker M, et al. Impulse oscillometry may be of value in detecting early manifestations of COPD. Respir Med 2012; 106: 1116-1123.

29 Oppenheimer BW, Goldring RM, Berger KI. Distal airway function assessed by oscillometry at varying respiratory rate: comparison with dynamic compliance. COPD 2009; 6: 162-170.

30 O'Shaughnessy TC, Ansari TW, Barnes NC, et al. Inflammation in bronchial biopsies of subjects with chronic bronchitis: inverse relationship of CD8+ T lymphocytes with FEV1. Am J Respir Crit Care Med 1997; 155: 852-857.

31 Fujimoto K, Yasuo M, Urushibata $\mathrm{K}$, et al. Airway inflammation during stable and acutely exacerbated chronic obstructive pulmonary disease. Eur Respir J 2005; 25: 640-646.

32 Medina-Contreras O, Geem D, Laur O, et al. CX3CR1 regulates intestinal macrophage homeostasis, bacterial translocation, and colitogenic Th17 responses in mice. J Clin Invest 2011; 121: 4787-4795.

33 McComb JG, Ranganathan M, Liu XH, et al. CX3CL1 up-regulation is associated with recruitment of CX3CR1 $1^{+}$ mononuclear phagocytes and $\mathrm{T}$ lymphocytes in the lungs during cigarette smoke-induced emphysema. Am J Pathol 2008; 173: 949-961.

34 Traves SL, Donnelly LE. Th17 cells in airway diseases. Curr Mol Med 2008; 8: 416-426.

35 Zhang L, Cheng Z, Liu W, et al. Expression of interleukin (IL)-10, IL-17A and IL-22 in serum and sputum of stable chronic obstructive pulmonary disease patients. COPD 2013; 10: 459-465.

36 Di Stefano A, Caramori G, Gnemmi I, et al. T helper type 17-related cytokine expression is increased in the bronchial mucosa of stable chronic obstructive pulmonary disease patients. Clin Exp Immunol 2009; 157: 316-324.

37 Saetta M, Di Stefano A, Turato G, et al. $\mathrm{CD}^{+}$T-lymphocytes in peripheral airways of smokers with chronic obstructive pulmonary disease. Am J Respir Crit Care Med 1998; 157: 822-826.

38 Miller M, Ramsdell J, Friedman PJ, et al. Computed tomographic scan-diagnosed chronic obstructive pulmonary disease-emphysema: eotaxin-1 is associated with bronchodilator response and extent of emphysema. J Allergy Clin Immunol 2007; 120: 1118-1125.

39 Joubert P, Lajoie-Kadoch S, Labonté I, et al. CCR3 expression and function in asthmatic airway smooth muscle cells. J Immunol 2005; 175: 2702-2708.

40 Hogg JC, Wright JL, Wiggs BR, et al. Lung structure and function in cigarette smokers. Thorax 1994; 49: 473-478. 